\title{
Erratum to: Chronic Cannabinoid Administration to Periadolescent Rats Modulates the Metabolic Response to Acute Cocaine in the Adult Brain
}

Alejandro Higuera-Matas, ${ }^{1,5}$ Maria Luisa Soto-Montenegro, ${ }^{2,3}$ Gonzalo L. Montoya, ${ }^{1,2}$ Verónica García-Vázquez, ${ }^{3}$ Javier Pascau, ${ }^{2}$ Miguel Miguéns, ${ }^{1}$ Nuria Del Olmo, ${ }^{4}$ Juan José Vaquero, ${ }^{2}$ Carmen García-Lecumberri, ${ }^{1}$ Manuel Desco, ${ }^{2,3}$ Emilio Ambrosio ${ }^{1}$

${ }^{1}$ Psychobiology Department, School of Psychology, UNED, C/Juan del Rosal nº10, 28040, Madrid, Spain

${ }^{2}$ Unidad de Medicina y Cirugía Experimental, Hospital General Universitario Gregorio Marañón, Madrid, Spain

${ }^{3}$ CIBERSAM, Madrid, Spain

${ }^{4}$ Department of Pharmaceutical and Food Sciences, School of Pharmacy, CEU University, Madrid, Spain

${ }^{5}$ Department of Pharmacology, School of Medicine, Universidad Complutense, Madrid, Spain

66 $V$ alues are expressed as mean \pm SEM and referred to total brain metabolism (100\%)" was inadvertently included in the caption to Figure 2.

The caption to Figure 2 should read:

Fig. 2. Interaction plots showing relative metabolism for the dorsal striatum of males (a) and the septal nuclei for females (b). Interactive effects between chronic periadolescent cannabinoid/vehicle exposure and adult cocaine or saline administration are shown.

The authors regret the error.

The online version of the original article can be found at http://dx.doi.org/ 10.1007/s11307-010-0388-8. 